\title{
The SEEMP Approach to Semantic Interoperability for e-Employment
}

\author{
E. Della Valle ${ }^{1}$, D. Cerizza ${ }^{1}$, I. Celino ${ }^{1}$, M.G. Fugini ${ }^{1}$, J. Estublier ${ }^{2}$ G. Vega ${ }^{2}$, M. Kerrigan ${ }^{3}$, A.Gómez \\ Pérez $^{4}$, J. Ramírez ${ }^{4}$ B. Villazón ${ }^{4}$, G. Zhao ${ }^{5}$, M. Cesarini ${ }^{6}$, and F. De Paoli ${ }^{6}$ \\ ${ }^{1}$ CEFRIEL - Politecnico of Milano, Via Fucini 2, 20133 Milano, Italy \\ ${ }^{2}$ Equipe Adele, LSR, Université Joseph Fourier,F-38041 Grenoble Cedex 9, France \\ ${ }^{3}$ STI Innsbruck, University of Innsbruck, Technikerstraße 21a, 6020 Innsbruck, Austria \\ ${ }^{4}$ Universidad Politécnica de Madrid, 28660 Boadilla del Monte, Madrid, Spain \\ ${ }^{5}$ Le Forem, Boulevard Tirou 104, 6000 Charleroi, Belgium \\ ${ }^{6}$ Università di Milano- Bicocca, Via dell'Innovazione, 20100 Milano, Italy
}

\begin{abstract}
SEEMP is a European Project that promotes increased partnership between labour market actors and the development of closer relations between private and public employment services, making optimal use of the various actors' specific characteristics, thus providing job-seekers and employers with better services. The need for a flexible collaboration gives rise to the issue of interoperability in both data exchange and share of services. SEEMP proposes a solution that relies on the concepts of services and semantics in order to provide a meaningful service-based communication among labour market actors requiring a minimal shared commitment.
\end{abstract}

\section{Current Situation}

European Member States have introduced major reforms to make the labour market more flexible, transparent and efficient, in compliance with the European Employment Strategy guidelines. Such major reforms include decentralization, liberalization of the mediation market (competition between public and private actors), and quality monitoring of Employment Service (ES) staff and services. As an effect, ESs understood the need for making available on-line a one-stop shop for employment. This results in an increased used of Information Communication Technology (ICT) and a boost in differentiating and personalizing the services they offer (e.g., Borsa Lavoro Lombardia, Le FOREM, EURES ${ }^{1}$, etc.).

The current employment market is characterized by high heterogeneity of models and actors; in particular we can distinguish between Public Employment Services (PES) and Private Employment Services (PRES) ${ }^{2}$. The ICT systems in ESs can serve different purposes: facilitating job-matching and job mobility for job seekers and employers; improving the functioning of labour markets; coordinating the exchange of information; allowing a more efficient management of ES internal services; monitoring of local market trends; personalized services, etc. The need for reconciling local, regional and national policies is increasing and it concerns the combination of services and data provided by different actors.

SEEMP project (IST-4-027347-STP) aims to design and implement a prototype of an Interoperability infrastructure for PESs and PRESs. More specifically, SEEMP is developing an EIFcompliant Architecture (EC, 2004) to allow collaboration between the employment services that exist in Europe. The resulting European Employment Marketplace will overcome the national barriers complying, at the same time, with the local policies of each Member State. Job-seekers and employers will have better services that operate at European scale thanks to SEEMP, which promotes increased partnership between labour market actors and the development of closer relations between private and public

\footnotetext{
${ }^{1}$ See respectively http://www.borsalavorolombardia.net/, http://www.leforem.be/ and http://europa.eu.int/eures/

${ }^{2}$ In the rest of the chapter we use ES when we refer both to public and private actors, whereas we us PES and PRES referring respectively to public and private actors.
} 
employment services. For instance, the matching of Job Offers and CVs across European borders will become possible, eventually increasing labour hiring and workforce mobility.

\subsection{Problem Statement}

In order to fulfil SEEMP's ambitious goals several problems must be solved at an organizational and technical level.

At an organizational level, the business model of SEEMP has to be catchy for all ESs. The main reason for an ES to buy in is by creating added value for its local users (both job seekers and employers) by offering interconnections with other ESs. Today it is normal for users to insert their CV or Job Offers into many ESs and collect, laboriously, the results by hand. With SEEMP in place, each ES will be able to collaborate with other ESs. From the perspective of the end user, the add-value is the outreach to other niches of the job market without 'being stretched out'. End users will be able insert their CV or Job Offer into one ES and collect pan-European results. From the ESs perspective it will result in an increase in both the number of users and their faithfulness to each ES, thus an increase in transaction volume.

At a technical level, the need for a flexible collaboration between ESs, gives rise to the issue of interoperability in both data exchange and share of services. The technical approach of SEEMP relies on the concepts of Web services and semantics. Web services, exploited in a Software Engineering manner, enable an easier maintenance of the integration. Semantics, encoded in the systems by the means of ontologies and mediators, allow for the reconciliation of hundreds of local professional profiles and taxonomies.

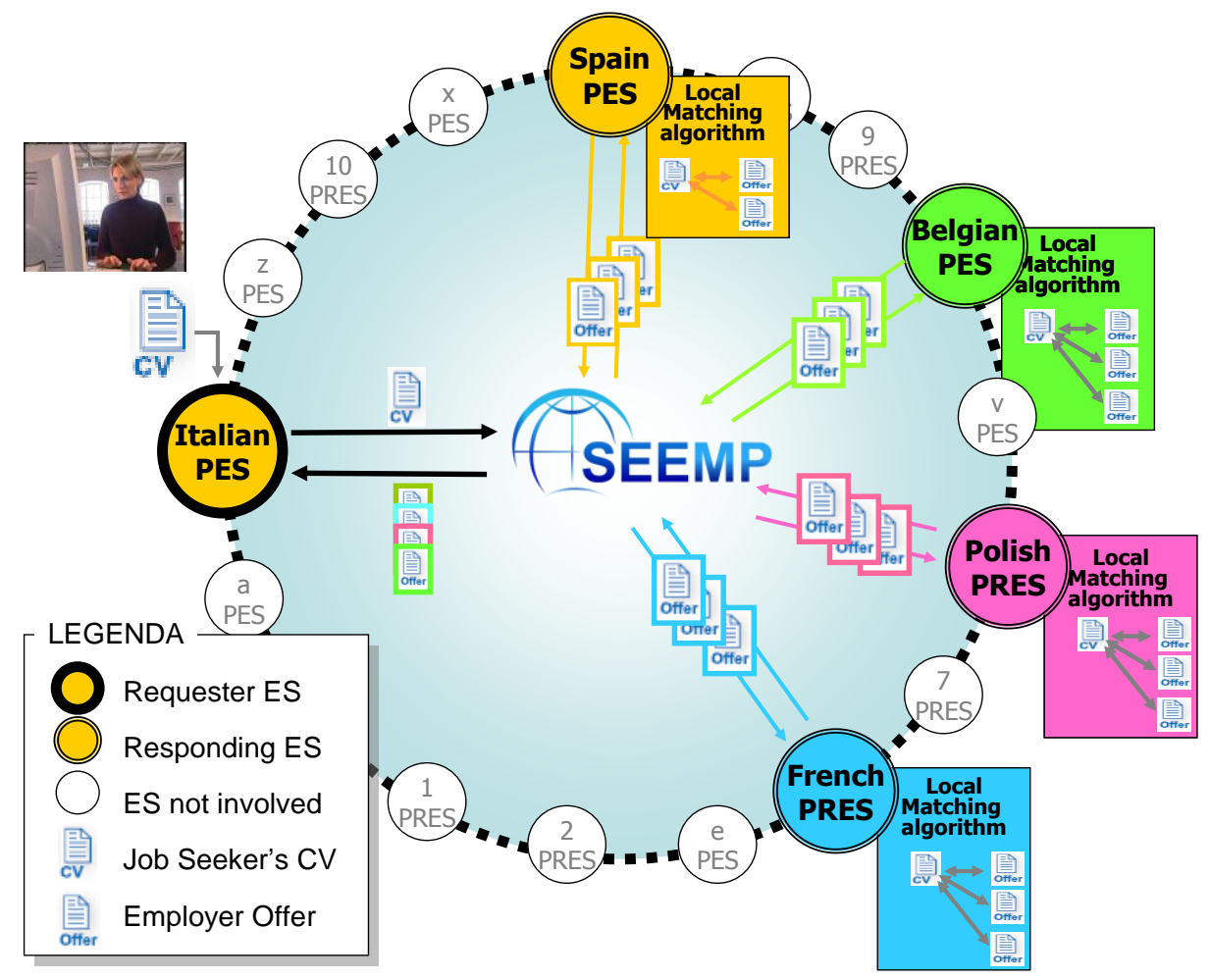

Fig. 1. The running example of distributed matching of CVs and Job Offers.

The SEEMP solution will expose, following the well established Software Engineering approach of Mélusine (Estublier, J., Vega, G., 2005), a single consistent set of abstract services each ES can invoke. Such abstract services will provide a multilateral interoperability solution that delegates the execution of the services to the local ESs (in accordance with the subsidiarity principle) and aggregates the results before sending the response back to the invoker. Moreover, following the innovative Web Service Modeling Ontology (WSMO) (Fensel et al. , 2006) approach, we will capture the semantics shared among ESs in a single consistent model. Such a model includes a reference ontology to which the local semantics is mapped, as well as a semantic description of the local Web services for their automatic use. A set of tools will be provided to each ES for modeling its local ontology and for mappings its local ontology to 
the reference ontology. As a technical result SEEMP will enable a meaningful service-based collaboration among ESs.

A e-Employment running example. For the discussion of this chapter we will consider a running example derived by the user requirements of the SEEMP project:

Job seekers (companies) put their CVS (Job Offers) on a local PES and ask to match them with the Job Offers (CVS) other users put in different PESS and PRESs through SEEMP.

It may look like a fairly simple example, but to reach its potential EU-wide audience, this eEmployment running example (see Figure 1) needs to fulfil a wider set of requirements than the respective local ES service. A local matching service is designed for national/regional requirements only (i.e., central database, single professional taxonomy, single user language, etc.). SEEMP has to be able to send the request, which an end-user submits to the local PES (the Italian ES on the left of Figure 1), to the other PESs and PRESs in the marketplace. In order to avoid asking "all" ESs, it has to select those that are most likely will be able to provide an answer and send the request only to them (the two PESs and the two PRESs on the right of Figure 1). Moreover, the answers should be merged and ranked homogeneously by the SEEMP before they are sent back to the local ES.

\subsection{Interoperability Issues}

The running example presented above highlights the need for a system that covers the whole EU and subsumes hundreds of real heterogeneous systems existing in many different EU countries and regions. It implies the resolution of:

- Language heterogeneity, e.g., an Italian Java Analyst Programmer may be looking for Job Offers written in all the different European languages;

- CVs and Job Offers structural heterogeneity, i.e. the use of standards like HR-XML is not wide spread and a multitude of local formats exists

- CVs and Job Offers content description heterogeneity, i.e., European level occupation classifications like ISCO-88 exist, but they do not reflect legitimate differences and perspectives of political economic, cultural and legal environments

- System heterogeneity in terms of service interface and behavior, i.e., no standard exists for eEmployment services thus each ES implements its own external interfaces and behaviour

These issues are typical interoperability issues that SEEMP helps to solve. The need for interoperability at European Level among e-Government services has been perceived since 1999 (1720/1999/EC) with the adoption of a series of actions and measures for pan-European electronic interchange of data between administrations, businesses and citizens (IDABC) (2004/387/EC). The main result of IDABC is the European Interoperability Framework (EIF) (EC, 2004). EIF follows the principle of subsidiarity in addressing the interoperability problem at all levels: organizational, semantic and technical. The principle of subsidiarity recommends that the internal workings of administrations and EU Institutions should not be interefered with. One crucial aspect, deriving from the principle of subsidiarity, is to keep responsibility decentralized; in other words each partner should be able to keep its own business process almost unchanged and to provide an external point for interacting with its processes, which EIF terms Business Interoperability Interfaces (BII). Quoting from IDABC: "it is unrealistic to believe that administrations from different Member States will be able to harmonize their business processes because of pan-European requirements". EIF itself does not prescribe any technical solution, but rather it recommends the principles to be considered for any e-Government service to be set up at a pan-European level, namely accessibility, multilingualism, security, privacy, use of open standards and of open source software (where possible) and, last but not least, use of multilateral solutions. SEEMP proposes itself as an implementation of EIF in the domain of e-Employment.

\section{Proposed Solution}

SEEMP relies on the concept of Service. Web services are quickly becoming the standard for Business to Business integration, allowing for loose, decoupled architectures to be designed and built. Following the EIF, each PES and PRES must expose its Business Interfaces as Web services. SEEMP uses Mélusine (Estublier, J., Vega, G., 2005) as a tool for modeling abstract services and orchestrating the process of 
delegating the execution to distributed independent service providers. SEEMP, as a marketplace, models a unique consistent set of Web services out of those exposed by the PESs and PRESs. Therefore the services exposed by SEEMP become the actual standard for the distributed independent service providers.

SEEMP relies on the concept of Semantics: Data semantics is captured in ontologies and the semantics of heterogeneity is captured in mediators. As for services, each local PES and PRES has its own local dictionary of terms and its own classification, which are regarded in SEEMP as a local ontology. Such an ontology provides a means for exchanging data (i.e., structure/content of the exchanged messages are classes/instances of such ontologies). All these ontologies differ but share a common knowledge about employment. SEEMP models a unique consistent ontology, called the Reference Ontology (RO), made up of those exposed by the PESs and PRESs. Therefore, the SEEMP RO becomes the actual standard for data exchanged between the PESs and PRESs. METHONTOLOGY (Gomez-Pérez, 2003) is employed as a methodology for developing and maintaining both the local and reference ontologies.

SEEMP combines Services and Semantics. The semantics of a given service is captured in semantic descriptions of Web services and user Goals; SEEMP adopts the Web Service Modeling Ontology (WSMO) (Fensel et al. , 2006) as the conceptual model for described Services and the data they exchange. WSMO is used to semantically describe the data model for data exchanged between SEEMP services, the SEEMP Web services exposed by ESs, the requirements of service requestors as Goals, and Mediators for resolving heterogeneity issues. The Web Service Modeling Language (WSML) (de Bruijn, 2006) provides a concrete syntax for encoding these semantic descriptions using the WSMO model.

\subsection{Minimal Shared Commitment}

In the domain of e-Employment, different Employment Services, both public and private, collect CVs and Job Offers in order to match demand with supply. Each ES covers either a region or an occupational sector. As a result, the employment market is severely fragmented and many ESs perceive the need of sharing information in order to provide a better service to their customers. However, they would never exchange CVs or Job Offers, since they contain sensitive information (like contact details). Instead, the ESs use "anonymized" versions of CVs and Job Offers, which we have named Candidacies and Vacancies, respectively. Therefore, if an ES exchanges a Candidacy/Vacancy with another ES, it potentially enlarges the possibilities of finding a match, without giving the peer the chance to by-pass it by directly contacting the Job Seeker/Employer.

The prerequisite of the employment domain can be generalized to a common industrial need: parties agree on general principles, but then they only commit to a subset of the possible implications of these principles and keep for themselves all their disagreements. Therefore, we draw the conclusion that the common notion of shared agreement is not enough to manage the complexity of the industrial scenarios we face. We believe that two important notions have to be made explicit: commitment and disagreement. It is worth noting that usually when parties provide ontology commitment the intended meaning is that all parties commit to the "entire" ontology. On the contrary, we propose to give a "subjective" meaning to commitment and disagreement, which does not presume a common knowledge (Lewis, 1969 and Aumann, 1976) among all the parties: two parties may commit to (or disagree with) different parts of the agreement.

In order to move from the problem statement to the solution conception, we need to find appropriate methods and technologies. We need conceptual elements to capture the notions of agreement, commitment and disagreement, to make them operational and to express the respective relations among them. Ontologies have been used and are good for formalizing and sharing the agreement. The notion of commitment is usually associated to the notion of ontology, and this is certainly true in the context of agent communication. In agent-based systems, all agents usually share a single ontology. The Semantic Web vision, however, foresees an ecosystem of ontologies, because of the very nature of the Web which is "fractal" (Berners-Lee, 1998). Ontologies can be co-invented, they can partially overlap and, in developing a new ontology, the importing of existing ones is encouraged (Shadbolt et al., 1998). We believe that the "practical" meaning of ontological commitment in the Semantic Web is slightly different from the original one. In formal terms, committing to an ontology that imports several other ones is the same as committing to one big ontology obtained by the union of all of them; however, in practical terms, committing to the ontology that includes the import annotations is partially an "unconscious" commitment, in that it means trusting the knowledge engineer who decided which ontologies to import. 
Therefore, our best practice is to distinguish between the RO, which captures the shared agreement, and the local ontologies, which captures the commitment and the disagreement of the various parties. We propose to build the RO including all the details that are needed to allow for a meaningful communication between each pair of parties, thus including also details that most of the parties would consider either inessential or not sharable. Then, each party can develop its local ontology, partially by importing parts of the reference ontology, and partially by developing its own point of view. Every time a local ontology imports a part of the RO, the party is considered to commit to the imported parts of the RO. Moreover, every time a part of the local ontology is aligned to a part of the RO (e.g., by the means of ontology-toontology mediators (de Bruijn et al., 2006b)), the party is also said to commit to that part of the RO. A particular attention should be given in capturing also the source of disagreement within the local ontology. Finally, each party should make available to all other actors the part of the local ontology that explains its different point of view without causing conflicts.

\subsection{The SEEMP Solution}

The SEEMP solution is composed of a reference part (all the dark components in Figure 2), which reflects the "minimal shared commitment" both in terms of services and semantics, and by the connectors toward the various local actors (the components in shading colors in Figure 2).

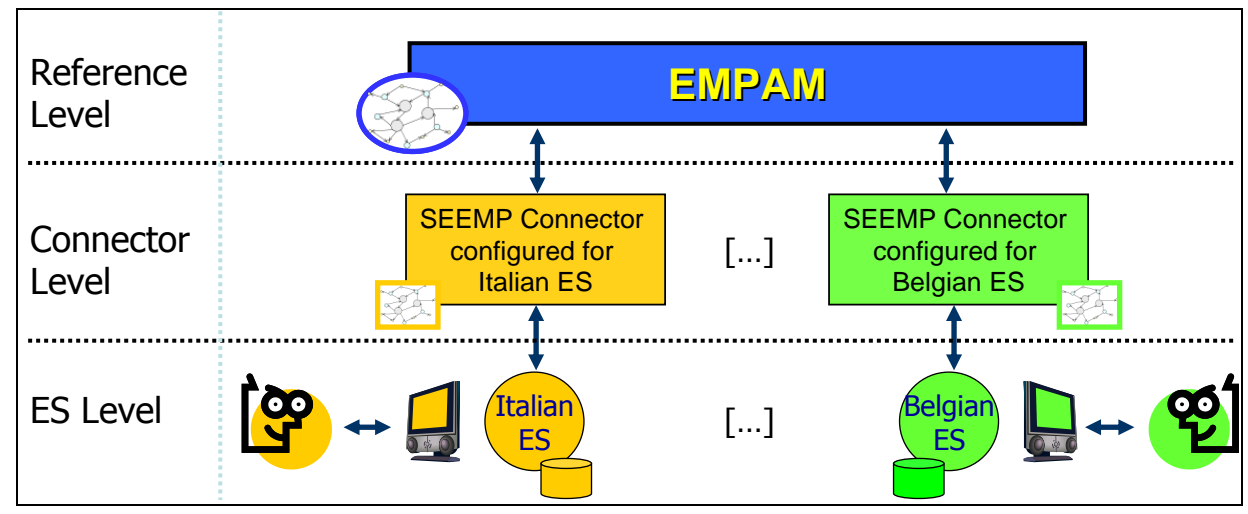

Fig. 2. An overview of the SEEMP solution

\subsubsection{Structural Overview}

The reference part of SEEMP solution is made up of the central abstract machine, named EMPAM (Employment Market Place Abstract Machine) and a set of SEEMP services.

The EMPAM is an abstract machine, in that it does not perform directly any operations, but rather offers abstract services that are made concrete by delegation: when the abstract service is invoked, the EMPAM delegates its execution to the appropriate ES by invoking the correspondent concrete services. It acts as a multilateral solution (as request by EIF), in which all the services connected to the EMPAM are made available to all other ESs, i.e. they ensure a pan-European level of services without interfering with the Business processes of each ES.

The SEEMP services are meant to support EMPAM execution. The running example requires two SEEMP services, namely discovery and ranking. The discovery service is offered by Glue (Della Valle et al., 2005). The EMPAM invokes Glue Discovery Engine before delegating the execution to the concrete services exposed by the ESs. Glue analyzes the CV sent by the invoking ES and it selects among all ESs those that are most likely to be able to return relevant Job Offers. The ranking service is invoked by the EMPAM after all the concrete services have answered and it merges the results providing a homogeneous ranking of the returned Job Offers. It also deletes duplicated Job Offers that are returned from different ESs.

The SEEMP connectors enable the collaboration that occurs between the EMPAM and a given ES. A SEEMP connector will exist for each of the ESs that are connected to the EMPAM and has two main responsibilities: 
- Lifting and Lowering: when communicating with the ES any outgoing (or incoming) data which is exchanged by the means of Web services must be lifted from the syntactic XML level to the semantic WSML level in terms of the local ontologies of the ES (or lowered back to the syntactic XML level from the semantic WSML level).

- Resolving Heterogeneity: each ES has its own local ontology that represents its view on the employment domain. The SEEMP connector is responsible for resolving these heterogeneity issues by converting all the ontologized data (the data lifted from the XML received from the ES) into data in terms of the reference ontology shared by all partners and vice versa.

\subsubsection{Functional Overview}

By combining the EMPAM and the connectors, the SEEMP solution enables a meaningful service-based collaboration among ESs. Figure 3 illustrates how such a meaning collaboration occurs when executing the example from section 1.1:

1. The user inserts a CV into the Italian PES and requests relevant Job Offers

2. The Italian ES invokes the matching service of the marketplace providing the $\mathrm{CV}$ in $\mathrm{XML}$

3. The SEEMP connector lifts the XML CV to the semantic level in terms of the Italian local ontology and then translates the CV from the Italian ontology to the reference ontology

4. The discovery service in the EMPAM analyzes the CV and selects among all ESs those that are most likely to be able to return relevant Job Offers

5. The EMPAM invokes in parallel the local matching service of the selected ESs

6. The various connectors translate the $\mathrm{CV}$ from the reference ontology to the local ontology (i.e., the Belgian and French ESs), lower the local ontology instances to the local ES XML format and invoke the local service with the $\mathrm{CV}$

7. The Belgian and French PESs match the provided CV with their local respository of Job Offers, with all the processing occuring locally, and return those that match to the connector

8. The respective connector lifts the Job Offers from XML to the local ontology and translates the Job Offers from each local ontology to the reference one

9. The ranking service in the EMPAM merges, at a semantic level, the responses and ranks the Job Offers homogeneously

10. The Job Offers are sent back in terms of the reference ontology to the Italian connector that translate them in the Italian ontology and lower them back to XML

11. The connector responds to the Italian ES

12. Finally the ES displays the Job Offers to the user.

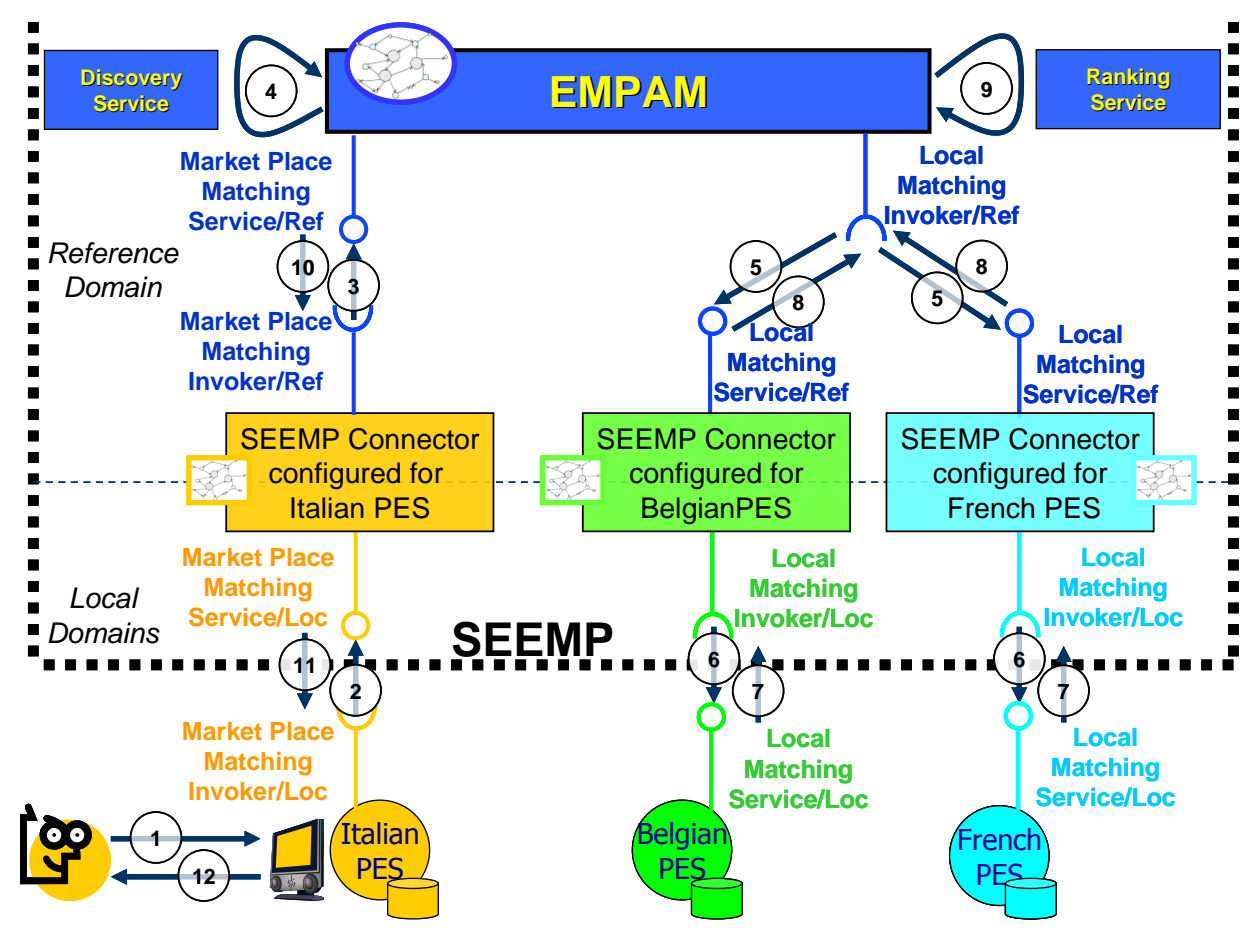

Fig. 3. How SEEMP solution enables meaningful service-based collaboration. 


\section{Solution Details}

\subsection{The SEEMP Reference Ontology for e-Employment}

The Reference Ontology (RO) is a core component of the system. It acts as a common "language" in the form of a set of controlled vocabularies that describe the details of the employment sector. The Reference Ontology has to be rich enough to support the semantic needs of all the Employment Services involved currently and in the future, i.e. it must be possible for an Employment Service to map a meaning full amount of its own Local Ontology to the Reference Ontology. The Reference Ontology also has to be a scalable, adaptable and maintainable ontology. For these reasons it was developed following the METHONTOLOGY approach (Gomez-Pérez, 2003).

The specification of the RO is based on international standards (like NACE, ISCO-88 (COM), FOET, etc.) and codes (like ISO 3166, ISO 6392, etc.). The RO is composed of thirteen sub-ontologies: Competence, Compensation, Driving License, Economic Activity, Education, Geography, Job Offer, Job Seeker, Labour Regulatory, Language, Occupation, Skill and Time. The main sub-ontologies are the Job Offer and Job Seeker ontologies, which are intended to represent the structure of a Job Offer and a CV respectively. While these two sub-ontologies were built starting from the HR-XML recommendations, the other sub-ontologies were derived from the available international standards/codes and ES classifications, chosen according to criteria that will be explained in the following of the chapter.

In order to choose the most suitable human resource management standards for modeling CVs and Job Offers, the degree of coverage needed in the desired domain has been considered, taking into account the scope and size of the standards; However, too broad a coverage may move us further away from the European reality; therefore we have tried to find a tradeoff between coverage and the following concerns:

- The Current European Need: it is important that the Reference Ontology focuses on the current European reality, because the Employment Services involved in SEEMP are European, and the Employment Marketplace will be used in a European context.

- Employment Service Recommendations: in order to assess the quality of the Reference Ontology, the opinion of existing Employment Services is crucial as they have a deep knowledge of the employment domain.

When choosing the standards, we selected the ICT domain as a "proof of concept" for prototyping the SEEMP marketplace. Hence, the chosen standards should cover the ICT domain to an acceptable degree. Narrowing the focus for prototype purposes results in a more focused Reference Ontology that can be validated more easily than modeling the entire employment domain, also modeling even a subsection of the employment involves the modeling of all primitives that will be needed across the entire domain. In the case of the Occupation ontology, for example, we have chosen one standard (ISCO-88 (COM)), but have also taken into account concepts coming from other classifications, in order to obtain a richer classification for the ICT domain.

When specifying Job Offers and CVs, it is also necessary to refer to general purpose international codes such as Country codes, currency codes, etc. For this aim, the chosen codes have been the ISO codes, enriched in some cases with classifications provided by individual Employment Services. Finally, the representation of Job Offers and CVs also requires temporal concepts such as Interval and Instant. So, in order to represent these concepts in the final RO, the DAML time ontology was chosen among the most relevant time ontologies found in the literature on ontologies.

\subsection{Developing Local Ontologies for SEEMP}

We propose two possible approaches to building the local ontologies for a given Employment Service:

1. Taking the Reference Ontology as a Seed: in this case, the concepts in the local ontologies are an extension in depth of the concepts already present in the RO; the consequence is that the effort required to transform instances between the Local and Reference Ontologies is low, while the complexity of translating between the local ontologies and the local schemata is higher

2. Reverse Engineering from ES Schemas: this is the easiest way for ontologizing the data of an ES, since each concept in a local ontology is the semantic expression of a relevant concept in the respective ES's schema; the consequence is that the translation between local ontologies and local 
schemata is not complex, while the creation of mappings between the Reference and Local ontologies can be difficult and costly to maintain

The suggested best practice is to use the first option when few ESs are present in the marketplace, and, when further ESs join the marketplace, to move progressively towards the second option. The balance between the two options is a trade off between allowing for meaningful communication and requiring minimal shared commitment.

Since each ES talks in its own "language", i.e. the local ontology that represents its view on the employment domain, its respective connector is responsible for resolving these heterogeneity issues, by translating the local content into the terms of the Reference Ontology. In this way, all the ESs in the marketplace speak the same language, and heterogeneity issues are resolved. Crucially each of the Employment servcies is responsible for managing its own mappings to the Reference Ontology, rather than managing mappings between every other possible local ontology in the SEEMP. Thus the addition of a new Employment Service to SEEMP represents no cost to exist members, reducing the barriers to entry for new ESs.

As a result of the introduction of a $\mathrm{RO}$, a set of local ontologies and the various ontology-toontology mediators it is possible to support scenarios in which parties agree while disagreeing (see section 5). For instance each European Country has its own regulation in terms of job legislation and skills/certifications required to apply for a job. This is especially relevant for some professions, such as physician, lawyers, teachers, and so on. Those regulations are mandatory for each Country, but, being "local", they can not fall within the shared agreement (i.e., the RO). As a concrete example, let us consider a Swiss ES and an Italian ES. Both express a positive commitment on the concepts related to jobs related to Academic Positions. However, the legislation about the prerequisites to apply for a University Professor position is different between Switzerland and Italy: the two Countries disagree on the necessity of holding a Ph.D. title. Therefore, the Swiss ES also makes explicit in its local ontology that each candidate for a Professor position should hold a Ph.D. title (whereas in Italy this is not mandatory).

\subsection{The Employment Market Place Abstract Machine (EMPAM)}

The EMPAM machine is implemented as a Mélusine application, which means it is structured following the Mélusine three layers approach (cf. Figure 4).

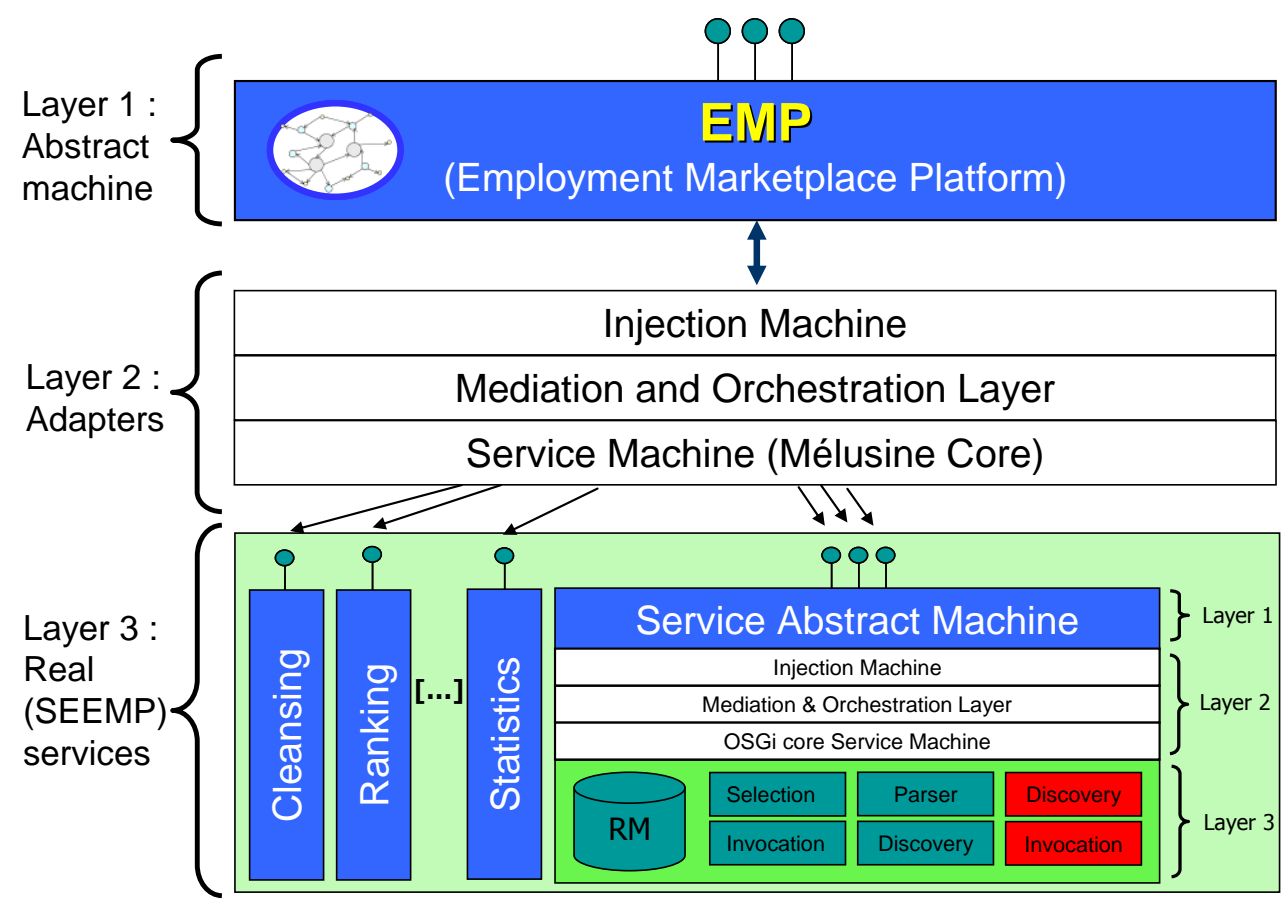

Fig. 4. The levels that make up the EMPAM as a Mélusine application. 
Layer 1 - Abstract Machine: The higher EMPAM machine layer is a Java abstract program where abstract classes represent the concepts present in our SEEMP public employment service. EMPAM acts as an ES covering the whole of Europe, i.e. it acts as if all the CV and vacancies were present in its repositories. However the EMPAM is abstract since, in fact, it does not have any information locally, but delegates the responsibilities of storing and matching CVs and Job Offers to real ESs. The EMPAM program defines functions like repository access and matching that are not implemented at all or are only sketches of the functions behaviour.

Layer 2 - Adapters: The responsibiliy of the second layer if to link the abstract services in the first layer with the real services provided by the Employment Services. To that end this layer is itself structured in three layers:

- The Injection Machine, whose duty is to capture those methods that need to be completed or implemented, and to transfer the call to the mediation and orchestration layer

- The Mediation and Orchestration Layer, which is responsible for transforming a single abstract method call into a potentially complex orchestration of real lower level services that together will perform the required function

- The Service Machine, whose duty is to transparently find and load the required SEEMP service and to call it. In SEEMP, this service machine is the core Mélusine service machine (an open source implementation of OSGi).

Layer 3 - SEEMP Services: SEEMP Services are OSGi services, and are called accordingly by Mélusine. Using OSGi ensures the optimal performance of the EMPAM, while leaving the system open to future extension with new SEEMP Services and even dynamic changes, such as the dynamic loading/unloading of services. Two classes of services have been identified:

- Services for Interacting with ESs: Most of the issues raised by EMPAM are related to discovering, selecting, parsing, and finally invoking a remote Employment Services connector. This functionality is delegated to a specific service, namely the Service Abstract Machine (SAM), which is itself a Mélusine application and therefore contains an abstract machine that defines the fundamental concepts and functions of a service machine. This layer is captured and delegated to an orchestration layer that calls local services, which in the scope of SEEMP, are WSMX components (Haller et al., 2005), wrapped as OSGi services.

- Services of the marketplace: These services include the cleansing, ranking and statistic functions, and will include, in the future, the implementation of specific functions and repositories of the EMPAM machine i.e. those functions and information not available in the ESs. Functions and information available in ESs are made available by calling the SAM service.

\subsection{SEEMP Connectors}

All communication between the EMPAM and a given ES occurs through the given ESs' SEEMP Connector and has two main responsibilities:

- Lifting and Lowering: Current Employment Services only deal in terms of structured XML content and do not deal in terms of ontologies. Within the SEEMP marketplace it is important that all content is ontologized so that it can be reasoned over, thus the SEEMP connector must lift all messages received from a given ES to the semantic level. This is done by converting the XML content received from the ES to WSML in terms of the local ontologies of the ES. Likewise when communicating with the ES, any outgoing data must be lowered back to the XML level so that the ES can understand the content. Since WSMO elements can be serialized in an RDF format, this task could be done by converting XML content to RDF first, and then converting RDF to WSML. In SEEMP this task is achieved by the means of an extension to the R2O language (Barrasa et al., 2004), which can be used to describe mappings between XML schemas and ontologies, and to its related processor ODEMapster (Rodriguez et al., 2006) .

- Resolving Heterogeneity: Each Employment Service has its own view of the employment domain. The SEEMP connector is responsible for resolving heterogeneity issues that may exist between a given ESs' view of the employment domain and the EMPAMs' view of the employment domain. This is achieved by transforming the data recieved from for the terms of the local ontology into the terms of the Reference Ontology. Of course when the EMPAM wishes to send data back to a given ES the reverse process must be performed such that data in the terms of the Reference Ontology is transformed into the terms of the ESs local ontology. 
As described in section 7.1 the Reference Ontology represents the bridge, or common vocabulary, that the ESs communicate through. Rather than managing mappings between every possible ontology pair, which essentially becomes unmanageable as the number of ESs in the markeplace grows, each ES need only maintain mappings to and from the reference ontology. These mappings represent a set of rules describing how to convert an instance from the local ontology to an instance of the reference ontology and vice versa. This process can be seen in Figure 5, when PES1 wishes to communicate with PES2 it is necessary to convert the message from PES1s' local ontology to the reference ontology and then to convert the message to PES2s' local ontology.

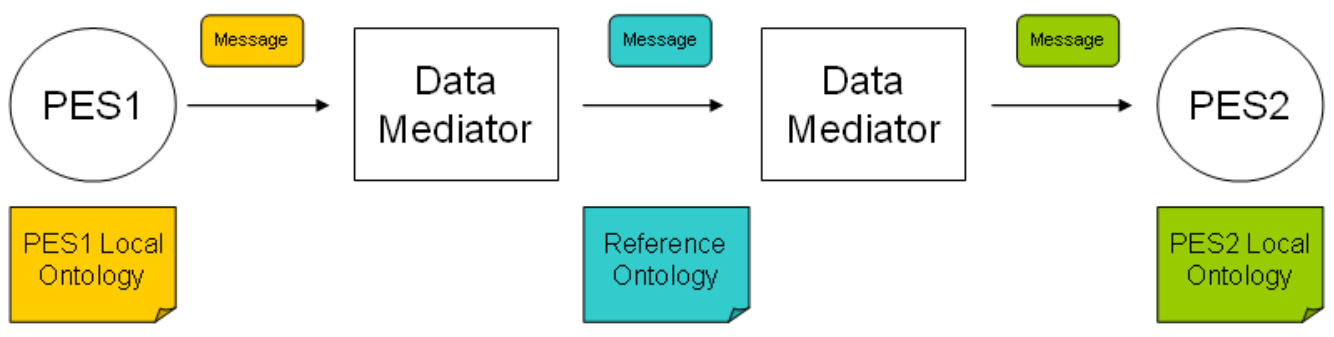

Fig. 5. Converting between two local ontologies via the reference ontology.

Technologically this is achieved using the WSMX Data Mediation (Mocan et al., 2006) . This work is made up of two components, the first being a design time component, within which the ES can describe the mappings between their local ontology and the reference ontology, and the second being a run time component, which is responsible for executing the mappings at run time to transform the messages between ontologies.

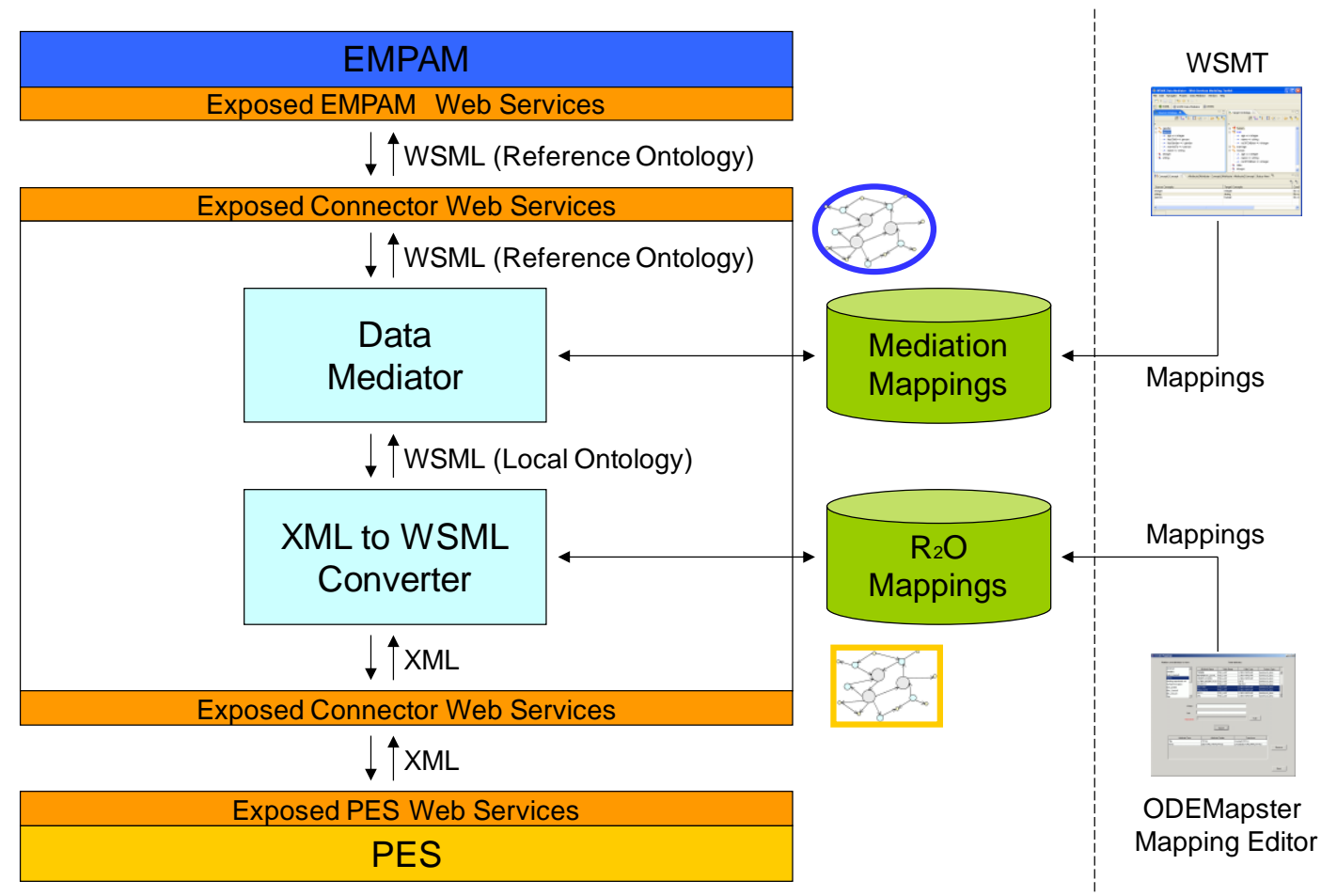

Fig. 6: The SEEMP Connector internal architecture.

A reusable SEEMP connector is built by bringing together the functionality described above. The architecture of the SEEMP Connector outlined in the Figure 6 shows the ES communicating with the connector using XML via the exposed web services. This XML is then lifted to the local ontology using the R20 mappings stored in the repository and furthermore converted to the reference ontology using the data mediation mappings. Ultimately the EMPAM is invoked using messages in the reference ontology via its exposed web services. Communication also occurs in the opposite direction.

Each of the ESs joining the marketplace will require its own SEEMP connector, however the only difference between any two connectors is the code for executing the ESs exposed Web services as each 
ES will expose services in a different way. The need for individual SEEMP connectors could be removed through the use of WSMO Choreography (Fensel et al. ,2006) to describe the interfaces of the ES services and the integration of the WSMX choreography engine (Cimpian et al., 2005) and invoker into the SEEMP Connector. This is considered as future work for the SEEMP Connector.

\subsection{A Running Example}

In Figure 7 we provide a screen-shot of the page receive when users of an Italian PES ask for SEEMP results. All information is presented in Italian even if they were originated by different ESs in different languages.

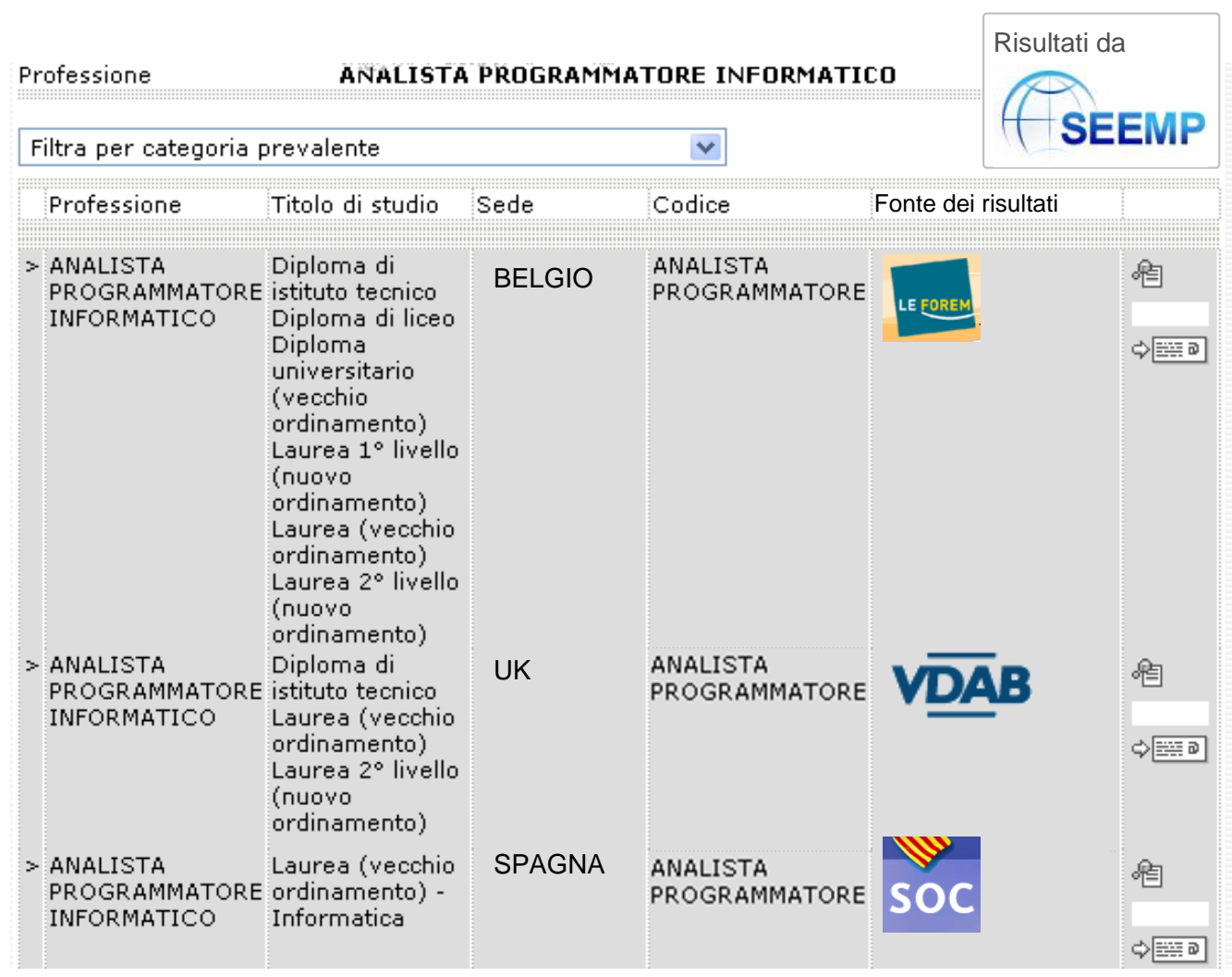

Fig. 7: How the Job Offers SEEMP got from al EU are presented to an Italian User

What happens "behind the scenes" (see Figure 8) is that the user submits their query with their profile, which is translated locally into a message with the local Italian code (e.g., Code X-it); afterwards, the Italian SEEMP Connector generates the respective ontological message, which refers to the local ontological concept (e.g., Concept_X-it) and then translate the message into the reference version, which contains indications about the respective reference ontological concepts (e.g., RefConceptY and RefConceptX). Once the message is expressed in the reference format, it can be sent to the other ESs, whose connectors can translate it into the local ontological format (e.g., Concept_Y-be) and then in the local codes (e.g., Code_Y-be), processable by the local system. The receiver ESs can finally send back their response messages, if any, by following the same process.

The result is that an Italian user that does not speak French can find a job that requires only English language skills in Belgium, from an ES that was not previously accessible to him because of the language used in their user interface. Moreover, he can access this information without the knowledge of the existence of the ES, without needing to subscribe directly to the other ESs system and without re-posting his profile. 


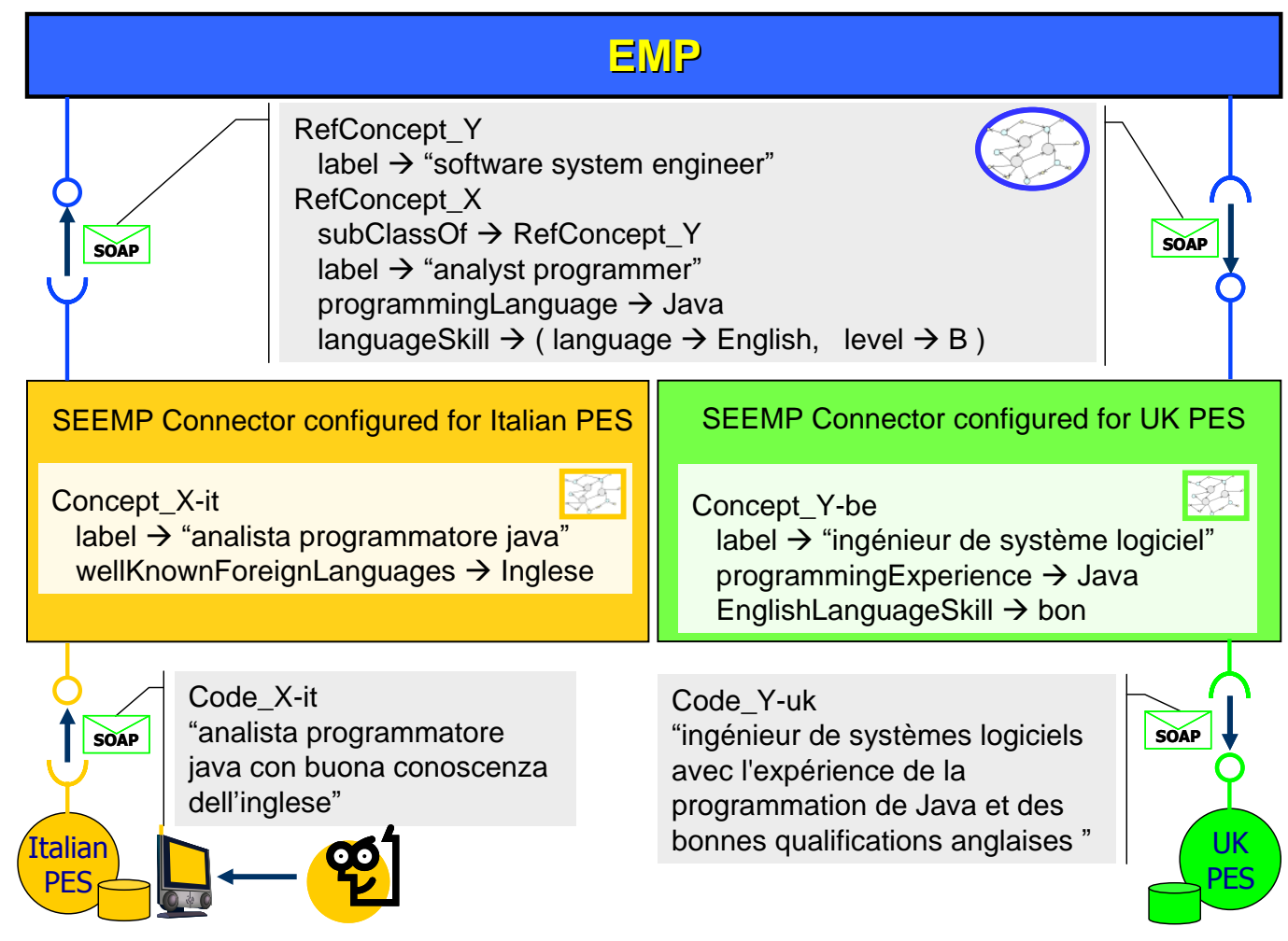

Fig. 8: Translated a message from one ES to another using Local and Reference Ontologies

\section{Alternatives}

In order to draw a comparison between SEEMP and other approaches we selected two case studies: private employment networks (e.g. Adecco) and hierarchical networks (e.g. Borsa Lavoro Lombardia, EURES). Moreover we consider the differences from the point of view of both the CEO and the CTO, i.e the decision makers and the IT experts.

Compared to other approaches the SEEMP solution offers the CEO a way to enforce the subsidiarity principle, therefore valuing the contribution of each ES to the marketplace. In private networks the subsidiarity principle is not applicable, while in hierarchical networks most of the nodes are passive actors. Moreover the marketplace creates added value by increasing the number of interconnections, hence resulting in more faithful users (more $\mathrm{JO} / \mathrm{CV}$ accessible using the user language) and in more transactions. Many Job Offers that can only be found today by inserting a CV into multiple ESs and gathering the results manually by hand, now become available through the interface of each ES., with no additional effort on behalf of the user.

For the CTO the SEEMP solution enables an easier maintenance of the integration with other ESs and minor integration costs. It has been proven that Web services used in a Service Oriented Architecture eases the effort of integration and maintenance. Moreover semantics makes the process of mapping different terminology easier because tools (such as the WSMT) can analyzed local and reference ontologies, by comparing sub-structures and by searching for synonyms, and can guide the IT Administrator in creating the mappings. Thank to this support, the mapping definition process requires less time or provides more precise mappings in the same amount of time.

Normally to achieve this benefit the CEO has to develop a "partnership", i.e., the ability to collaborate with other peers, ES or staffing industries. The partnerships are different in the two case studies. In private network everything is agreed in advance. In hierarchical network partnership are necessary, but no peer to peer decision taking is possible. Decisions are institutionally imposed top-down. Moreover SEEMP supports the CTO by providing a comprehensive set of tools and methodologies for service and semantic interoperability.

Concerning services the CTO has to expose his ES APIs as Local Web services and has to provide support for invoking EMPAM services. However, they don't have to understand interfaces and behavior 
of other ES (as in hierarchical solutions) because the connector presents the market place as if the ES was invoking its own services. Concerning semantics the CTO has to model his data structures and content and has to defining mappings with the Reference Ontology, but as discussed above, this is made easier through the provision of good tool support.

What has to be built, and which SEEMP alone is anable to achieve, is a comprehensive reference ontology and abstract service machine that encompasses the entire employment domain. Developing and maintenance this reference part of SEEMP is not a ICT problem; it is a matter of reaching agreement at organizational level. As already discussed in section 4 the goal of SEEMP is reaching a "minimal shared commitment" in which ESs agree on high-level aspects, allowing for collaboration among them, while disagreeing on minor details that differentiate one ES from the others.

\section{Cost and Benefits}

As described in section 2.1, there is a minimum level of commitment required from a given ES if they wish to join the SEEMP network of Employment Services. This commitment is design to ensure that the joining of a new ES has no negative impact on existing ESs within the network and the commitment is kept to a minimal level to ensure that barriers to entry for new ES are as low as possible. The SEEMP architecture revolves around two main themes, namely Services and Semantics, and there exists a minimum commitment with respect to both of these themes. As can be seen in section 3, the SEEMP architecture is broken up into three levels and here we are concerned with the interaction between the EMP and a given ES through the Connector. The EMP assumes that each ES exposes a standardized set of services that can be invoked to achieve some specified functionality. The EMP also assumes that all data exchanged by these services are ontological data, formalised in the SEEMP Reference Ontology. Therefore we can define the minimum level of commitment for a given ES as the functionality that it needs to provide so that the assumptions, upon which EMP is based, are true.

At the ES level, each employment service will exist in its own non standardised world, with each ES having a different level of technological advancement in terms of how data is stored and exchanged with outside parties (if external sharing of data happens at all). The process of meeting the minimum level of commitment in terms of Service and Semantics should not involve any change to the internal processes of the employment service (unless they choose to make such a change). Thus the SEEMP Connector is designed as a mechanism to layer on top of the existing architecture of the ES and provide the services expected by the EMP that exchange data in terms of the SEEMP Reference Ontology. A SEEMP Connector is thus a piece of software that exposes the services expected by the EMP and communicates in terms of instances of the reference ontology.

There are two main tasks that must be undertaken by the engineer wishing to build a connector between the Web services exposed by the ES and the Web services expected by the EMP. Firstly, the engineer must identify the mapping between the ESs services and those expected by the EMP and link these together via some code. In other words when the EMP invokes the matchCV interface of the connector, this invocation must result in the invocation of one or more of the interfaces of the ES. Of course the reverse mapping must also be made such that invocations from the ES are translated into invocations to the EMP. Thus the connector must expose services upwards towards the EMP and downwards towards the ES. While the services exposed to the EMP are standardized, the choice of the services to expose to the ES is at the discretion of the connector architecture. However, it is recommended to mirror the services that the ES itself already exposes, such that the ES views the connector as if communicating with itself as is shown in Fig 8-a. Such an approach to exposing services to the ES ensures the minimal change to the functionality of the ES when it wishes to invoke the connector and ensures that ES engineers, who are already familiar with their own interfaces, can successfully work with the connector services with minimum effort. Secondly the XML data exchanged by the ES Web services must be lifted to the instances of the reference ontology in order to meet the minimum commitment of semantics. There are a number of approaches to performing this lifting:

Direct mapping of XML to the reference ontology. Performing this step involves the use of technologies like XSLT or $\mathrm{X}_{2} \mathrm{O}$ Mappings to define a set of syntactic mappings between the XML received from the ES to the instances of the reference ontology expected by the EMP and vice versa. Integrating this approach with Figure 9-a results in an architecture like that depicted in Figure 9-b. This approach is only be feasible in cases where the XML data is semantically very close to the reference ontology. In cases where a big gap between the two formats exists, the mappings would quickly become 
too complicated for such a simple approach. If an ES decides to adopt this type of connector, the following figure reports on an estimation of the integration costs to be sustained.

The solution based on the Syntactic Connector is very simple at the beginning since it doesn't require any particular know-how or deep understanding of the interoperability problem. The development may become harder than expected but acceptable in those situations where the XML data is semantically very close to the reference ontology. However, with this solution their is a risk of increased cost during the maintenance phase and, in particular, it's infeasible when important changes in the ES are required (see Figure 9).

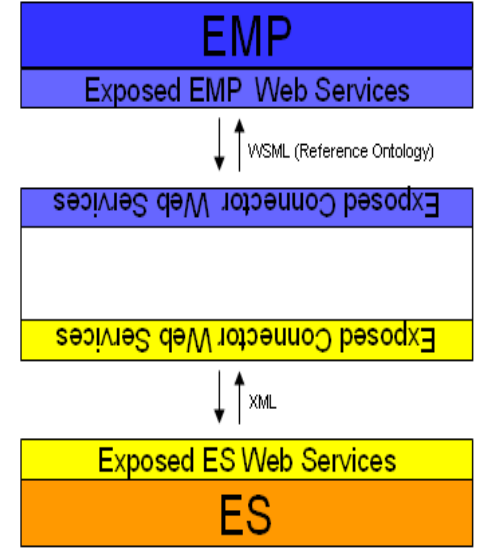

a) SEEMP connector interfaces

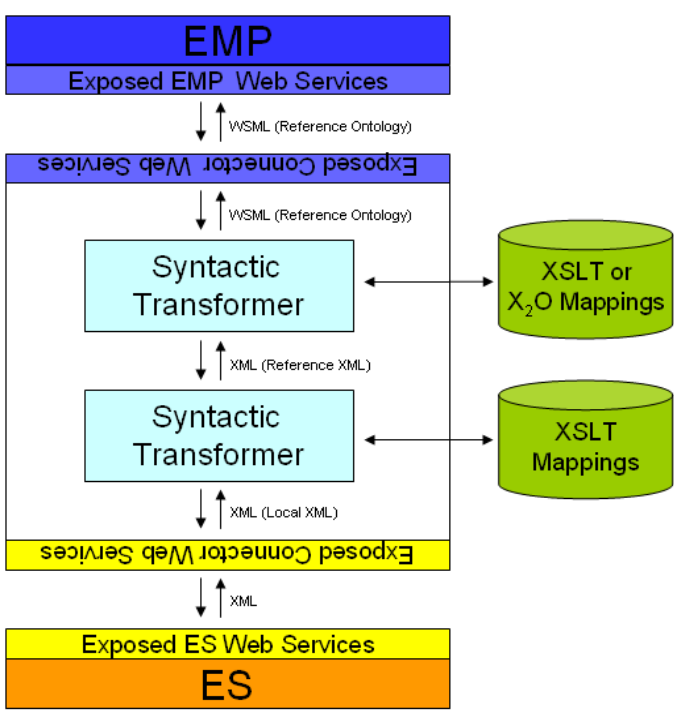

c) A Two-step syntactic connector

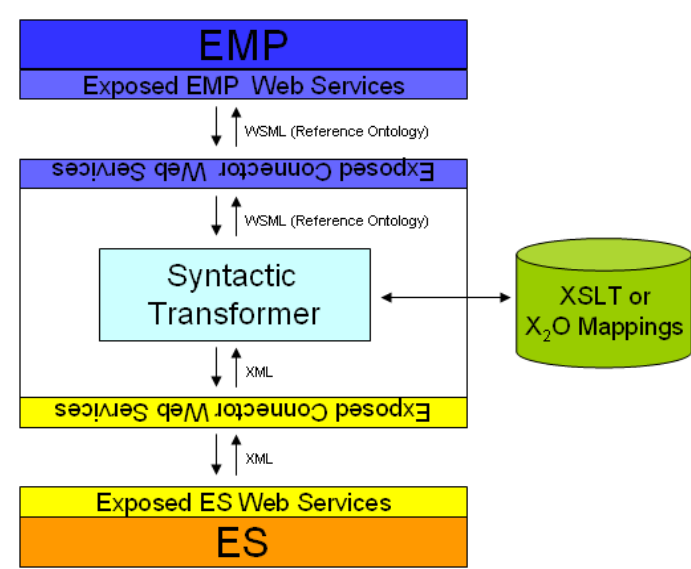

b) A Syntactic connector

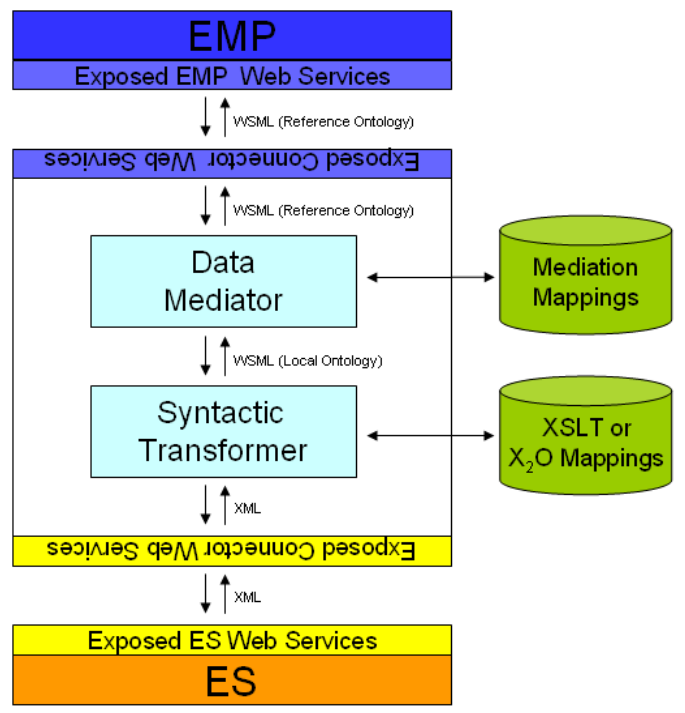

d) A Two-step semantic connector

Fig. 9: Architecture of the possible SEEMP connectors.

Two step Syntactic XML translation. To break the problem into a more manageable one, an additional XML format can be included in the translation from local XML format to the reference ontology. This second XML format would be very similar to the semantics of the reference ontology allowing for the complexity of the mappings to be broken into two steps. The translation from the Local XML format to the new XML format would resolve semantic issues between the two XML formats (although it should noted that this would be done syntactically via a technology like XSLT) and the second step of lifting the new XML format to the reference ontology would be relatively trivial, achieved using a syntactic transformation. Again with this approach a large difference between the two XML formats in terms of semantics would result in large amounts of mappings being created and resulting in the need to maintain these mappings as the XML formats and reference ontologies evolve. Integrating this approach with Figure 9-a results in an architecture like that depicted in Figure 9-c. 


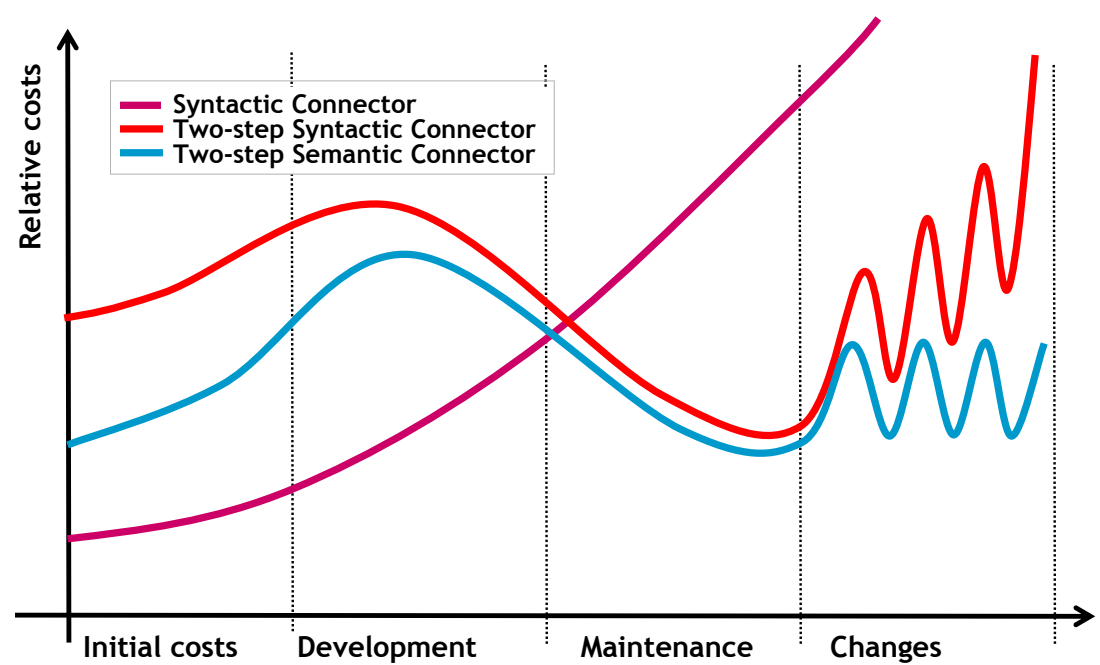

Fig. 10: Estimating integration costs for the possible SEEMP connectors.

The initial costs and the development are higher than the previous solution (see Figure 10). This is because this solution requires the engineer to understand the problem related to the heterogeneity gap early in the process (both syntactic and semantics) and to develop a syntactic transformation between the local XML and the reference XML. However, thanks to a clear separation between these gaps, this solution promises to be more flexible in terms of maintenance and changes. Nevertheless, whenever the changes become critical, the integration costs may become more expensive.

Two-step Semantic XML translation. One of the major problems with the previous designs, especially in cases where the Employment Services schemas are semantically very different from the Reference Ontology, is that they try to use syntactic technologies to resolve a problem with semntics and this results in the creation of many complex mappings that are difficult to create and harder to maintain. The inclusion of a semantic transformation step in the process helps alleviate this problem as semantic transformation involves fewer and less complicated mappings. In this method a local ontology is built that represents the local ESs view of the employment domain. The Local XML received from the ESs Web services is translated to the local ontology using XSLT or $\mathrm{X}_{2} \mathrm{O}$ mappings. The remaining problem to be solved is the transformation of the instances of the local ontology to instances of the reference ontology, which can be solved by technologies such as the WSMX Data Mediation component with the accompanying design time environment in the Web Service Modeling Toolkit (WSMT) (Kerrigan et al. , 2007). This approach provides the cleanest and most maintainable solution for performing the translation of local XML to instances of the reference ontology, however it does have the overhead of maintaining the ES local ontology. Integrating this approach with Fig 8-a results in an architecture like that depicted in Figure 9-c.

In the three above approaches to translation two specific technologies are mentioned, namely $\mathrm{R}_{2} \mathrm{O}$ mappings for translating from XML to Ontologies and WSMX Data Mediation for performing the transformation of instances of one ontology into instances of another ontology.

This solution follows a progress similar to the previous architecture and it's slightly cheaper in the initial phases since the SEEMP technology is shipped with a specific tool for designing mappings. Moreover, thanks to the fact that the tool can leverage the semantic expressiveness for creating mappings, this solution promises to be more flexible in the face of changes without increasing the cost when the changes become critical. For example, in the case that the local ontology will evolve by detailing some parts and the reference ontology remains stable in those parts, the semantic mappings will not be affected thanks to the subsumption features.

\section{Risk Assessment}

A SWOT analysis (see Table 1) for SEEMP was performed in order assess strengths, weaknesses, opportunities and threats of the marketplace. In the rest of the chapter we gave a detailed explanation of the benefits of SEEMP. The most remarkable weaknesses of SEEMP are related to costs. 


\begin{tabular}{|c|c|}
\hline $\begin{array}{l}\text { Strengths } \\
\text { - No competitors; } \\
\text { - Respond to a need from current market } \\
\text { agencies ( Public + Private) by increasing: } \\
\text { - Geographical scope in an extending } \\
\text { EU; } \\
\text { - The quality through mapping } \\
\text { technologies based on ontologies and } \\
\text { web services }\end{array}$ & $\begin{array}{l}\text { Weaknesses } \\
\text { - Entrance costs; } \\
\text { - Kick-off costs } \\
\text { - Management of semantic protocols }\end{array}$ \\
\hline \begin{tabular}{l}
\multicolumn{1}{c}{ Opportunities } \\
- Enlargement of the EU; \\
- Increasing of regions prerogatives regarding \\
the employment policy ( no more national \\
only anymore); \\
- Obligation for PES to respond to programs \\
like : e-administration; modernisation of \\
PES,... \\
- Mobility framework at EU level \\
- Shortage of skills with highly mobile \\
profiles
\end{tabular} & - Laws regarding privacy \\
\hline
\end{tabular}

Table 1. SEEMP SWOT analysis

The initial costs for starting the SEEMP community would be considerable in relation to the actual benefits. However, these benefits are essential to the public sector and EC actors so SEEMP be targeted first to Public Sector stakeholders and to educational providers. We consider that the first experimentations of SEEMP are relevant for a pool of Public actors, with a few selected pool of private actors, e.g., some large companies for example operating in the ICT market.

Once a SEEMP community exists, it would be much easier to attract private services and provide better ROI reports. However, even for the public integration with the private sector is necessary and thus even at the initial stage SEEMP should incorporate in the business plans the private sector.

\section{Conclusions}

This chapter presented the SEEMP approach in supporting meaningful servicebased collaboration among public and private employment services. The following results have been shown:

- Services and Semantics are the key concepts for abstracting from the hundreds of heterogeneous systems already in place that are evolving separately. They provide a straightforward way to implement the subsidiarity principle of EIF.

- Tthe combination of an abstract service machine with a Reference Ontology is a technically sound Multi-lateral approach for marketplace implementation. Each actor in the marketplace has to care only about integrating with the marketplace. The marketplace will offer services to support the interaction with other actors.

- A mix of Software Engineering and a Semantic approach is required to achieve flexibility. The two approaches nicely complement each other. By means of "conventional" software engineering design SEEMP built an abstract machine that can run on "conventional" technology and at the same time embeds semantics both in the form of ontologyies and mediators, and in the form of semantic-aware components (i.e. ODE mapster, WSMX data mediation, Glue).

Currently the SEEMP consortium is running a pilot that shows the integration of EURES and Borsa Lavoro Lombardia. This integration has so far allowed for the testing of the functional aspects of SEEMP approach. The next step is integrating with the Le FOREM ES as a validation case. We expect, the reference ontology and the abstract machine to be so well designed that Le FOREM introduction would have no impact on the existing services. 
Future work includes extending the number of abstract services included in the EMPAM and the respective concepts in the reference and local ontologies. For instance, one essential service of SEEMP should be the possibility to have regularly (monthly, weekly, daily, . . . ) a set of key indicators regarding labour market in all participant regions (job seekers, Job Offers, training, etc.), in a common and comparable language, both in terms of methods (definitions, calculation of indicators, etc.) and in terms of technical requirements.

\section{Acknowledgments}

This research has been partially supported by the SEEMP EU-funded project (IST-4-027347-STREP). We thank all the colleagues that work in SEEMP for the for the fruitful discussions that lead to this work.

\section{References}

1720/1999/EC. Decision of the European Parliament and of the Council of 12 July 1999

2004/387/EC. Decision of the European Parliament and of the Council on Interoperable Delivery of panEuropean Services to Public Administrations, 2004

Aumann, R.J. (1976). Agreeing to Disagree. The Annals of Statistics 4(6) 1236-1239

Barrasa, J., Corcho, O., Gomez-Pérez, A. (2004). R2O, an extensible and semantically based databasetoontology mapping language. In: Second International Workshop on Semantic Web and Databases.

Berners-Lee, T (1998). The Fractal Nature of the Web, working draft.

Cimpian, E., Mocan, A. (2005). WSMX Process Mediation Based on Choreographies. In: Business Process Management Workshops. 130-143

de Bruijn, J., Ehrig, M., Feier, C., Martìns-Recuerda, F., Scharffe, F., Weiten, M. (2006b). Ontology Mediation, Merging, and Aligning In: Semantic Web Technologies: Trends and Research in Ontologybased Systems. John Wiley \& Sons, Ltd. 95-113

de Bruijn, J., Lausen, H., Polleres, A., Fensel, D. (2006). The Web Service Modeling Language: An overview. In: Proceedings of the 3rd European Semantic Web Conference (ESWC2006), Budva, Montenegro, Springer-Verlag

Della Valle, E., Cerizza, D. (2005). The mediators centric approach to automatic web service discovery of glue. In: MEDIATE2005. Volume 168 of CEUR Workshop Proceedings., CEUR-WS.org 35-50

EC (2004). European Communities: European interoperability framework for pan-european egovernment services. Technical report, Office for Official Publications of the European Communities.

Estublier, J., Vega, G. (2005). Reuse and variability in large software applications. In: ESEC/SIGSOFT FSE. 316-325

Fensel, D., Lausen, H., Polleres, A., de Bruijn, J., Stollberg, M., Roman, D., Domingue, J. (2006). Enabling Semantic Web Services - The Web Service Modeling Ontology. Springer

Gomez-Pérez, A., Fernandez-Lopez, M., Corcho, O. (2003). Ontological Engineering. Springer Verlag

Haller, A., Cimpian, E., Mocan, A., Oren, E., Bussler, C. (2005). A Semantic Service-Oriented Architecture. In: ICWS. 321-328

Kerrigan, M., Mocan, A., Tanler, M., and Fensel, D. (2007). The Web Service Modeling Toolkit - An Integrated Development Environment for Semantic Web Services (System Description), Proceedings of the 4th European Semantic Web Conference (ESWC2007), June 2007, Innsbruck, Austria

Lewis, D. (1969). Convention: A Philosophical Study. Oxford: Blackburn

Mocan, A., Cimpian, E., Kerrigan, M. (2006). Formal model for ontology mapping creation. In: International Semantic Web Conference. 459-472

Rodriguez, J.B., Gomez-Pérez, A. (2006). Upgrading relational legacy data to the semantic web. In: WWW '06: Proceedings of the 15th international conference on World Wide Web, New York, NY, USA, ACM Press 1069-1070 
Shadbolt, N., Berners-Lee, T., Hall, W. (2006). The Semantic Web Revisited. IEEE Intelligent Systems 21(3) 96-101

\section{Additional Reading}

IDABC and Capgemini (2004). Architecture for delivering pan-European e-Government services

E. Della Valle, M.G. Fugini, D. Cerizza, I. Celino, P. Guarrera, G. Zhao, G. Monteleone, A. Papageorgiou, J. Estublier, J. Ramìrez, B. Villazon, M. Kerrigan (2007) SEEMP: A marketplace for the Labour Market - In Proceedings of e-challenges 2007, 24 - 26 October 2007, The Hague, The Netherlands

E. Della Valle, D. Cerizza, and I. Celino, J. Estublier, G. Vega, M. Kerrigan, J. Ramirez, B. Villazon, P. Guarrera, G. Zhao and G. Monteleone (2007) SEEMP: an Semantic Interoperability Infrastructure for egovernment services in the employment sector - In Proceedings of 4th European Semantic Web Conference, ESWC 2007, LNCS 4519, Innsbruck, Austria

E. Della Valle, D. Cerizza, and I. Celino, J. Estublier, G. Vega, M. Kerrigan, J. Ramirez, B. Villazon, P. Guarrera, G. Zhao and G. Monteleone (2007) SEEMP: Meaningful Service-based Collaboration Among Labour Market Actors - In Proceedings of 10th International Conference on Business Information Systems, BIS 2007, LNCS 4439, Poznan, Poland 